\title{
The War against Problematic Biofilms in the Oil and Gas Industry
}

\section{Dake $X \mathbf{u}^{1}$ and Tingyue $\mathbf{G u}^{2^{\star}}$}

${ }^{1}$ Institute of Metal Research, Chinese Academy of Sciences, Shenyang, Liaoning Province, China

${ }^{2}$ Department of Chemical and Biomolecular Engineering, Institute for Corrosion and Multiphase Technology, Ohio University, Athens, OH 45701, USA

Biocorrosion (also known as microbiologically influenced corrosion or MIC) results in billions of dollars in damages each year in the United States alone [1]. They affect many industries such as the oil and gas industry, water utilities and power plants. Biogenic reservoir souring and biofouling are also major issues in the oil and gas industry. Microbial biofilms are often the culprit [2]. They are becoming increasingly problematic due to widespread practice of enhanced oil recovery usually in the form of water injection. Fresh water is not used in oil production because it is a scarce resource. Instead, seawater is often injected in addition to produced water extracted from the reservoir during oil production. Seawater brings nutrients (e.g., organic carbons) and oxidants (e.g., sulfate). It also brings various microbes including sulfate reducing bacteria (SRB). Oxygen is typically removed from seawater by using nitrogen gas stripping and oxygen scavengers. To prevent MIC and reservoir souring, seawater is sometimes dosed with biocides. Huge amounts of biocides are needed because the volume of treated seawater is massive. For example, Saudi Aramco's Qurayyah Seawater Treatment Plant has a capacity of 14 million barrels of treated seawater for oil production throughout the kingdom.

In the downhole environment, SRB respiration of an organic carbon such as acetate with sulfate as the terminal electron acceptor produces hydrogen sulfide known as sour gas. This process provides energy for SRB metabolism. Biogenic reservoir souring by this corrosive and highly toxic gas is mitigated by periodical biocide dosing [3]. To mitigate biofilms, biocides are dosed together with other chemicals such as corrosion inhibitors for $\mathrm{CO}_{2}, \mathrm{H}_{2} \mathrm{~S}$ corrosion, oxygen scavengers and scale removers in pigging operations [4]. Biocides are also used in the hydraulic fracturing fluids for shale gas and oil production to prevent the biofouling that plugs fissures [5].

It is well known that biofilm cells (i.e., sessile cells) are much more difficult to kill than planktonic cells because biofilms possess various defense mechanisms [6]. Dense biofilms are diffusional barriers that slow down the penetration of biocides. Sessile cells in biofilms can deliberately slow down their metabolic rates to reduce the intake of harmful chemicals. They can upregulate genes that code for proteins that weaken or destroy biocides. They have various efflux pumps to pump out toxic chemicals from their bodies. The most remarkable defense mechanism is the formation of the called "persister cells." A biofilm can selectively preserve some tough cells during a biocide attack. The regrowth of the persister cells after the stress is removed has been found to be phenomenal once the biocide stress is removed [7]. A rule of thumb is that sessile cells require 10 times or higher biocide concentrations to treat compared to planktonic cells. In a human body, a bacterial infection can be treated with antibiotics. With the help of the human immune system, the bacterium that causes the infection can be completely eradicated. A field system is more difficult to rid of microbes completely. The selection of biocides is limited because of economic and environmental factors. Biocide concentrations and residence times are often limited such that a complete kill is untenable. There is a lack of immune system to help eradicate the residual bacteria. Even if a complete kill were achieved, it would not mean much if microbes are reintroduced due to fluid flow anyway. The inability for a complete kill inherently means that a biocide treatment will always promote the survival of resistant microbes. This is why biocide treatment runs in repeated cycles. A more efficacious biocide will reduce the sessile cell concentration to one or more log reductions, thus prolonging the gap between treatment cycles. Repeated biocide dosing promotes biocide resistance, eventually leading to biocide dosage escalation. For example, in oilfield operations, the initial dosage of tetrakis hydroxymethyl phosphonium sulfate (THPS), a green biocide popularly used in many oilfield operations, may be 50 or $100 \mathrm{ppm}(\mathrm{w} / \mathrm{w})$. Years later, 500 or even $1,000 \mathrm{ppm}$ would be needed due to biocide resistance. A high biocide dosage is not only expensive, but also causing environmental concerns when it is discharged. A high THPS dosage such as 1,000 ppm introduces so much sulfate in the drilling fluid, it starts to precipitate the barium ion in the fluid and results in scale formation that hampers the operation. Some operators are alarmed by the biocide escalation and they have started in earnest to find solutions because an established biocide has to be phased out.

Because of cost, environmental regulation, broad spectrum efficacy requirement, operator safety, chemical compatibility with other chemicals and other issues, only a few biocides are used in largescale oil and gas operations. THPS and glutaraldehyde have been the overwhelmingly dominating players. Unfortunately, there have been no blockbuster new biocides that can even marginally compete with them in the past few decades since the first reported use of THPS as an industrial biocide in 1983 [8]. Although researchers have been working hard to create more environmentally friendly biocides, a new blockbuster biocide is unlikely going to appear any time soon. New strategies are needed to solve the pressing issue of biocide resistance and dosage escalation in the war against problematic biofilms in the oil and gas industry. Biocide cocktails are usually not suitable because there are not many biocides to choose from in large-scale oil and gas operations. Cost and chemical compatibility are also major concerns. Bacteriophages have been proposed for SRB biofilm treatment [9]. However, they may be limited to certain microbial species. Surfactants or dispersants have long been used to help distribute biocides to the wall surfaces (instead of bulk fluid) where biofilms are. Some surfactants are biocidal [10]. Surfactants such as sodium dodecyl sulfate and Triton $\mathrm{X}-100$ can also weaken bacteria cell walls and thus making them more susceptible to biocides [10]. This relatively mature approach has had many years to perfect already. A new development in recent years is to use biocide enhancers other than surfactants and dispersants. The biocide enhancers themselves may be non-biocidal, but they make the sessile cells more vulnerable to biocides. The lack of toxicity actually

*Corresponding author:Tingyue Gu, Department of Chemical and Biomolecular Engineering, Institute for Corrosion and Multiphase Technology, Ohio University, Athens, OH 45701, USA, Tel: +1 740-593-1000; E-mail: gu@ohio.edu

Received September 02, 2015; Accepted September 04, 2015; Published September 09, 2015

Citation: Xu D, Gu T (2015) The War against Problematic Biofilms in the Oil and Gas Industry. J Microb Biochem Technol 7: e124. doi:10.4172/1948-5948.1000e124

Copyright: @ $2015 \mathrm{Xu} \mathrm{D}$, et al. This is an open-access article distributed under the terms of the Creative Commons Attribution License, which permits unrestricted use, distribution, and reproduction in any medium, provided the original author and source are credited. 
means that a biocide enhancer may encounter fewer discharge problems. Chelators such as ethylenediamine-N,N'-disuccinic acid (EDDS) are capable of making bacteria more vulnerable to antimicrobial attacks. However, their effective concentrations are rather high in the mitigation of oilfield biofilms [11]. A biocide enhancer with a low concentration may come from signaling molecules. Quorum-sensing inhibitors are too species-specific and prohibitively expensive. They may find eventual successes for medical biofilms, but unlikely for industrial biofilms. New classes of biocide enhancers are needed.

D-alanine appears as the terminus in the stem peptide of the peptidoglycan molecules in the cell walls of all bacteria. Lam et al. suggested that $\mathrm{D}$-amino acids might be a common way of self-adjustment used by cells to adapt to their changing environment [12]. KolodkinGal et al. speculated that D-alanine might be a signal molecule and its substitution by another $\mathrm{D}$-amino acid signals biofilm disassembly [13]. They showed that D-tyrosine, D-leucine, D-tryptophan, and D-methionine triggered the dispersal of bacterial biofilms of Bacillus subtilis, Staphylococcus aureus and Pseudomonas aeruginosa. Their lab experiments also found that a mixture of these D-amino acids was more effective than individual D-amino acids. For Desulfovibrio vulgaris (ATCC 7757), a recalcitrant SRB strain routinely used in MIC lab investigations, $\mathrm{Xu}$ et al. found that $1 \mathrm{ppm} \mathrm{D}$-tyrosine alone and 100 ppm D-methionine alone were both ineffective [6,14] against its biofilm. However, when a biocide stress in the form of $50 \mathrm{ppm}$ THPS was applied, they reduced the sessile cell counts $3 \log$ more than using 50 ppm THPS alone $[6,14]$. They argued that perhaps recalcitrant biofilms require a biocide stress to reinforce the message of biofilm dispersal. Individual bacteria may be susceptible to different $\mathrm{D}$-amino acids. An oilfield biofilm consortium tested by Li et al. was completely resistant to the attack by $50 \mathrm{ppm}$ THPS [15]. They found that $1 \mathrm{ppm}$ D-tyrosine and $100 \mathrm{ppm}$ D-methionine both failed to enhance the efficacy of $50 \mathrm{ppm}$ THPS. However, a mixture of D-tyrosine, D-leucine, D-tryptophan, and D-methionine with a total concentration of $50 \mathrm{ppm}$ achieved $4 \mathrm{log}$ reductions of the sessile cell counts in both the biofilm prevention test and the removal of pre-established biofilm test. The exact mechanism for D-amino acid enhancement of THPS is still unknown. Instead of the aforementioned D-alanine substitution hypothesis, Leiman et al. pointed out that $\mathrm{D}$-tyrosine, $\mathrm{D}$-leucine and $\mathrm{D}$-tryptophan inhibited the formation of the B. subtilis biofilm because they interfered with protein synthesis [16]. Problematic biofilms in the oil and gas industry may contain other microbial species such as archaea and fungi. However, if the bacterial component in a biofilm is prone to the D-amino acid enhanced biocide attack, the entire biofilm is weakened because biofilm recalcitrance comes from synergy within the community.

D-amino acids are now considered ubiquitous in nature. A significant fraction of $\mathrm{L}$-amino acids is converted to $\mathrm{D}$-amino acids when proteinaceous food products are heated or exposed to strong alkali for a long period of time [17]. D-amino acids routinely exist in animal and human diets [18]. They enter the aquatic and soil environments because rumen microorganisms secrete them and they end up in cattle wastes [18]. The use of low concentrations of naturally occurring D-amino acids to enhance a green biocide such as THPS may be an attractive approach in environmentally sensitive applications.

The biocide enhancer approach is one of the practical strategies that can be used to slow down the biocide dosage escalation problem in the war against biofilms. Apart from using microbe secreted chemicals, additional biocide enhancers may come from chemicals secreted by plants and animals, some of which have remarkable abilities to keep their surfaces free from biofilm buildups.

\section{References}

1. Walsh D, Pope D, Danford M, Huff T (1993) The effect of microstructure on microbiologically influenced corrosion. JOM 45: 22-30.

2. $\mathrm{Xu} \mathrm{D,} \mathrm{Gu} \mathrm{T} \mathrm{(2014)} \mathrm{Carbon} \mathrm{source} \mathrm{starvation} \mathrm{triggered} \mathrm{more} \mathrm{aggressive}$ corrosion against carbon steel by the Desulfovibrio vulgaris biofilm. Int Biodeter Biodegr 91: 74-81.

3. Gieg LM, Jack TR, Foght JM (2011) Biological souring and mitigation in oil reservoirs. Appl Microbiol Biotechnol 92: 263-282.

4. Falck C, Kleppe T, Maribu J (1997) Commissioning of long subsea pipelines environmental aspects (a report), Statoil, Norway.

5. Aminto A, Olson MS (2012) Four-compartment partition model of hazardous components in hydraulic fracturing fluid additives. J Nat Gas Sci Eng 7: 16-21.

6. Xu D, Li Y, Gu T (2012) A synergistic D-tyrosine and tetrakis hydroxymethy phosphonium sulfate biocide combination for the mitigation of an SRB biofilm. World J Microbiol Biotechnol 28: 3067-3074.

7. Costerton JW (2007) The biofilm primer, (1 ${ }^{\text {st }}$ edn) Springer-Verlag Berlin Heidelberg, Berlin-New York.

8. Jones C, Downward B, Edmunds S, Curtis T, Smith F (2012) THPS: A review of the first 25 years, lessons learned, value created and visions for the future. Nace International, Houston, TX.

9. Summer JS, Summer NS, Janes C, Liu M, Gill JJ, et al. (2011) Phage of sulfate reducing bacteria isolated from high saline environment. NACE International.

10. Chen X, Stewart PS (2000) Biofilm removal caused by chemical treatments Water Res 34: 4229-4233.

11. Wen J, Zhao K, Gu T, Raad II (2009) A green biocide enhancer for the treatment of sulfate-reducing bacteria (SRB) biofilms on carbon steel surfaces using glutaraldehyde. Int Biodeter Biodegr 63: 1102-1106.

12. Lam H, Oh DC, Cava F, Takacs CN, Clardy J, et al. (2009) D-amino acids govern stationary phase cell wall remodeling in bacteria. Science 325: 1552 1555

13. Kolodkin-Gal I, Romero D, Cao S, Clardy J, Kolter R, et al. (2010) D-amino acids trigger biofilm disassembly. Science 328: 627-629.

14. Xu D, Li Y, Gu T (2014) D-methionine as a biofilm dispersal signaling molecule enhanced tetrakis hydroxymethyl phosphonium sulfate mitigation of Desulfovibrio vulgaris biofilm and biocorrosion pitting. Mater Corros 65: 837-845.

15. Li Y, Zhang P, Xu D, Xu C, Gu T (2015) D-amino acids enhanced biocide mitigation of field biofilm consortia in lab tests, NACE International.

16. Leiman SA, May JM, Lebar MD, Kahne D, Kolter R, et al. (2013) D-amino acids indirectly inhibit biofilm formation in Bacillus subtilis by interfering with protein synthesis. J Bacteriol 195: 5391-5395.

17. Man EH, Bada JL (1987) Dietary D-amino acids. Annu Rev Nutr 7: 209-225.

18. Friedman M (2010) Origin, microbiology, nutrition, and pharmacology of D-amino acids. Chem Biodivers 7: 1491-1530. 